\title{
The Impact of Gd-Eob-Dtpa-Enhanced MR Cholangiography in Biliary Diseases: Comparison with T2-Weighted MR Cholangiopancreatography
}

\author{
Evrim Özmen ${ }^{1}$, Oktay Algın², Şehnaz Evrimler ${ }^{2}$, Halil Arslan $^{2}$ \\ ${ }^{1}$ Department of Radiology, İstanbul University Cerrahpaşa School of Medicine, İstanbul, Turkey \\ ${ }^{2}$ Department of Radiology, Atatürk Training and Research Hospital, Ankara, Turkey
}

Background: Contrast enhanced magnetic resonance cholangiography is a novel technique and promising method in demonstrating biliary tree anatomy and evaluating biliary disorders. However, to date, there are a limited number of studies that have focused on the impact of this technique.

Aims: We aimed to evaluate the additional role of contrast enhanced MR cholangiography (MRC) and compare contrast enhanced MRC with T2-weighted (w) magnetic resonance cholangiopancreatography (MRCP) in the diagnosis of biliary disorders.

Study Design: Diagnostic accuracy study.

Methods: The T2w-MRCP and contrast enhanced MRC sequences of 31 patients whose gold standard test results were available were scored visually for the existence of pathological findings with regard to any of the biliary diseases. Gadolinium ethoxybenzyl diethylenetriamine pentaacetic acid (Gd-EOB-DTPA) was used as the contrast agent. The correlation values were determined according to the statistical analysis made from those scores and the sensitivity, specificity and accuracy values of each sequence were detected as well.
Results: We detected that the correlation values with gold standard methods of contrast enhanced MRC sequences were significantly higher than the ones of T2wMRCP sequences. The correlation ratios of T2w-MRCP sequences were between 26 and $34 \%$, while those for contrast enhanced MRC sequences were between 81 and $83 \%$ for the first reader and the correlation ratios of T2w-MRCP sequences were between 10 and $61 \%$, whereas those of contrast enhanced MRC were between 79 and $81 \%$ for the second reader The mean sensitivity, specificity and accuracy values of T2w-MRCP sequences were $14.3-42.5 \%, 85-89.2 \%$ and $59.3-72.5 \%$, respectively, while the mean sensitivity, specificity and accuracy values of contrast enhanced MRC sequences were $100 \%, 86.7 \%$ and $93.2-93.3 \%$, respectively.

Conclusion: We suggest that obtaining of contrast enhanced MRC sequences in addition to the T2w-MRCP can be useful in the diagnosis of many diseases in relation with biliary tree.

Keywords: Cholangiopancreatography, contrast enhanced MRC, gadolinium ethoxybenzyl diethylenetriamine pentaacetic acid, magnetic resonance
Although ultrasonography (US) and computed tomography (CT) are fast and practical, they are often insufficient in the diagnosis of many biliary disorders. The interventional techniques including percutaneous transhepatic cholangiography (PTC) and endoscopic retrograde cholangiopancreatography (ERCP) are known as gold standard methods in the diagnosis of biliary diseases and can be used for treatment as well. Nev- ertheless these techniques are invasive, operator-dependent and also they include various complications.

In many cases, T2-weighted $(\mathrm{w})$ magnetic resonance cholangiopancreatography (MRCP) could provide an accurate diagnosis without the need for invasive diagnostic procedures. It does not involve any radiation risk, does not include any complications and does not require anesthesia. It can also be used

This study was presented as an electronic poster at the European Congress of Radiology, 6-10 March 2014, Vienna, Austria.

Address for Correspondence: Dr. Evrim Özmen, Department of Radiology, İstanbul University Cerrahpaşa School of Medicine, İstanbul, Turkey Phone: +90 5063668817 e-mail: evrimkilicdr@gmail.com

Received: 14 November $2014 \quad$ Accepted: 3 June $2015 \cdot$ DOI: 10.5152 /balkanmedj.2016.140872

Available at www.balkanmedicaljournal.org 
in episodes of pancreatitis or cholangitis, and presents images on different planes as well. Although T2w-MRCP could be an answer for many indications related to biliary diseases, there are some special circumstances that require contrast enhanced MRC, including the detection of biliary leakage and functional evaluation of the biliary system (1-4).

To the best of our knowledge, there are only limited published reports that have focused on the impact of contrast enhanced MRC in the evaluation of biliary diseases and variations (5-9). Those studies were achieved mostly with Gd-BOPTA

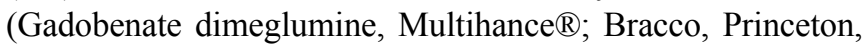
$\mathrm{NJ}$ ) and Mn-DPDP (Mangafodipir trisodium, Teslascan $®$; Nycomed Amersham, Princeton, NJ). Gd-EOB-DTPA (gadoxetic acid, Eovist ${ }^{\circledR}$ or Primovist $\AA$; Bayer Healthcare, Wayne, NJ), which was placed on the market in recent years, could provide optimal biliary system visualization with fewer doses than GdBOPTA (10). Also, it could supply earlier imaging for biliary tract than Gd-BOPTA (11). In this study, we used Gadolinium ethoxybenzyl diethylenetriamine pentaacetic acid (Gd-EOBDTPA) as a contrast agent and aimed to compare contrast enhanced MRC with T2w-MRCP, and to detect the correlations of both of these techniques with gold standard tests.

\section{MATERIALS AND METHODS}

\section{Study group}

Ethics committee approval was obtained for this prospective study. T2w-MRCP and contrast enhanced MRC examinations were applied to 54 patients between the ages of 24 and 89, with the mean age of 55.3, who were referred to the radiology department of our hospital over a period of 8 months with the prediagnosis of biliary disease. The study protocol was explained in detail to all of the patients participating in the study and informed consent was obtained from all patients. Gd-EOB-DTPA $\left(0.05 \mathrm{~mL} / \mathrm{kg}\right.$; Gadoxetic acid, Primovist ${ }^{\circledR}$, Bayer-Schering, Germany) was used as a contrast agent for contrast enhanced MRC. Twenty three of the 54 patients, whose ERCP, PTC or surgical and pathologic results could not be obtained, were excluded from the study. Thus, the remaining 31 patients were included in the study. Twenty of the 31 patients were men and 11 of them were women. Unconscious and non-cooperative patients, pregnant women, those with an allergy history, claustrophobic patients, those with implanted devices incompatible with MR such as pacemakers and those with impaired liver or kidney function were not included in the study.

\section{MRI protocol}

All of the MR images were obtained with a 1.5 Tesla MR Unit (Achieva; Philips Healthcare, Best, Holland), by using a
TABLE 1. MR imaging protocol of the study

\begin{tabular}{lccccc}
\hline $\begin{array}{l}\text { Imaging } \\
\text { Parameters }\end{array}$ & $\begin{array}{c}\text { T2w- } \\
\text { BTFE }\end{array}$ & $\begin{array}{c}\text { T2w- } \\
\text { TSE }\end{array}$ & $\begin{array}{c}\text { T2w- } \\
\text { SPAIR }\end{array}$ & $\begin{array}{c}\text { 3D- } \\
\text { heavily T2w }\end{array}$ & $\begin{array}{c}\text { CE- } \\
\text { T1w }\end{array}$ \\
\hline TR (ms) & 3.6 & 950 & 728 & 1204 & 3.1 \\
TE (ms) & 1.78 & 80 & 80 & 650 & 1.46 \\
Flip Angle & 90 & 90 & 90 & 90 & 10 \\
FOV & $330 \times 330$ & $330 \times 330$ & $330 \times 330$ & $256 \times 205$ & $330 \times 330$ \\
Matrix & $252 \times 242$ & $280 \times 275$ & $280 \times 275$ & $260 \times 260$ & $200 \times 180$ \\
$\begin{array}{l}\text { Slice thickness } \\
\text { (mm) }\end{array}$ & 6 & 6 & 6 & 0.8 & 2.5 \\
Time (sec) & 17 & 30 & 30 & 327 & 16 \\
$\begin{array}{l}\text { Acquisition } \\
\text { plane }\end{array}$ & Coronal & Axial & Axial & Coronal & $\begin{array}{c}\text { Axial- } \\
\text { coronal }\end{array}$ \\
\hline
\end{tabular}

FOV: field of view; BTFE: balanced turbo field echo; TSE: turbo spin echo; SPAIR: spectral adiabatic inversion recovery; $\mathrm{CE}$ : contrast enhanced; TR: time of repetition; TE: time of echo

phased-array body coil. All images were obtained by using the parallel imaging [sensitivity encoding (SENSE)] technique. All of the patients were asked to fast for at least 5-6 hours before the examination in order to prevent gallbladder contraction. T2w-MRCP and contrast enhanced MRC examinations were performed in supine position.

Acquisition protocol of MRC examinations are summarized in Table 1. The acquisition of three-dimensional (3D) heavily $\mathrm{T} 2 \mathrm{w}$ data was performed using respiratory triggering. Maximum intensity projection (MIP) images of 3D contrast enhanced $\mathrm{T} 1 \mathrm{w}$ sequences and heavily $\mathrm{T} 2 \mathrm{w}$ sequences were created with the non-commercial software that presents on MR devices following the acquisition of source images.

Contrast enhanced MRC images were obtained on arterial, venous and equilibrium phases after the intravenous administration of Gd-EOB-DTPA with the same parameters. The total MR examination time was about 25 minutes per patient. Although it depends on the prediagnoses of the patients, post-contrast $\mathrm{T} 1 \mathrm{w}$ images at the $20^{\text {th }}$ min (earlier phase) and $60^{\text {th }}$ min (later phase) were obtained again. In patients with a suspicion of gallbladder disease, post-contrast $\mathrm{T} 1 \mathrm{w}$ images were also obtained at $120^{\text {th }} \mathrm{min}$ in order to enable filling of the gallbladder with contrast agent. Those additional sequences which were obtained for late-phase contrast enhanced MRC images took approximately 5 minutes.

\section{Image analysis}

After MRC examinations, the images of all patients were loaded in the picture archiving and communications system (PACS). The demographic data and protocol numbers of all patients were recorded to the "MS excel, v. 2010" datasheet by the study coordinator who had 8 years of experience on MRC. The patients were randomized and collected in a table 
and given to the two observers who had experiences of 5 and 3 years respectively on MRC. These observers evaluated all of the MRC images independently and were unaware of the clinic, laboratory and surgery findings. In addition, the observers reevaluated the same images at least 3 weeks after the first evaluation for intraobserver reliability analysis.

T2w-MRCP images including T2w-balanced turbo field echo (T2W-BTFE), T2w-turbo spin echo (T2W-TSE), T2wspectral adiabatic inversion recovery (T2W-SPAIR), 3D heavily T2w sources and heavily T2w-MIP sequences and contrast enhanced MRC images including post-contrast T1w sequences in axial-coronal planes and post-contrast T1w MIP images were scored visually as follows in terms of the existence of biliary diseases; Score 0: No pathologic finding suggestive of biliary disease, Score 1: Some suspicious findings in favor of the investigated biliary disease, but these findings are insufficient for the diagnosis, Score 2: Adequate findings in favor of suspected biliary disease for the diagnosis.

In addition, consensus, which was scored by evaluating all sequences together, was included in statistical analyses. After the scoring procedure mentioned above, the two observers who made the scoring and the study coordinator performed an agreement meeting. In this meeting, MRC images, the findings of clinic-laboratory, gold standard methods (ERCP, PTC, and/or surgical \& pathological results), and/or clinical follow up results of the all of the patients were evaluated together. The final diagnosis of the patients included in the study was also made together. All of the findings, especially the results of the gold standard methods, were considered together for the final diagnosis.

\section{Statistical analysis}

Statistical analyses were performed with the program of Statistica (version 8.0, Statsoft Inc; Tulsa, Oklahoma, USA). The sensitivity, specificity and accuracy values of the sequences were determined. In correlation analysis of the sequences, the correlation percents of sequences with the results of the gold standard tests were detected with Spearman Rank Order Correlations test. In this test, a $p$ value below 0.05 was accepted as statistically significant. In addition, interobserver correlations and intraobserver reliabilities were calculated with Fleiss's kappa (к) coefficient. Intraand interobserver reliability ratios were expressed with the $\kappa$ coefficient. The $\kappa$ coefficient value is ordinarily between 0 and 1. " 1 " shows a complete agreement since " 0 " shows no agreement or agreement by chance (12). The $\kappa$ value should be interpreted within the clinical framework. However, a $\kappa$ value below 0.20 shows slight agreement, a $\kappa$ value between 0.21-0.40 shows fair agreement, a $\kappa$ value between 0.41 0.60 shows moderate agreement, a $\kappa$ value between 0.61 -
0.80 shows substantial agreement and a $\kappa$ value between 0.81-1.00 shows almost perfect agreement (13).

\section{RESULTS}

\section{Findings of the gold standard methods}

Fourteen of the 31 patients who were included in the study had no finding in favor of biliary disease on MRC images. For the remaining 17 patients, there was gallbladder perforation in 3 , acute cholecystitis in 3 , malign biliary obstruction in 3 , choledochal cyst in 2, bilioenteric fistula in 1, biliary stricture due to surgery in 1 , biliary system variation in 1 , biliary leakage due to surgery in 1, choledocholithiasis in 1 and von Meyenburg disease in 1 patient. In 3 patients in whom malign biliary obstruction was detected, there was an ampullary tumor in 1 , pancreas cancer in the head of the pancreas in 1 and Klatskin tumor in 1 patient. The patients with acute cholecystitis and gallbladder perforation were operated upon and their diagnoses were proven with histopathological examination results. The patients with bilioenteric fistula and choledocolithiasis were operated upon and the diagnoses were confirmed with operation results. The diagnoses of von Meyenburg disease and malign biliary obstructions were also confirmed with histopathological examination results after biopsy. PTC procedure was performed in 3 patients with malign biliary obstruction and ERCP procedure was applied in 3 patients who had stricture due to surgery, biliary system variation and biliary leakage, respectively. The interval between MRC examination and PTC or ERCP was less than or equal to 1 month.

In three patients with acute cholecystitis, we could not visualize the passage of contrast agent to the gallbladder in the $120^{\text {th }}$ min and the operation and pathology results of those patients were compatible with acute cholecystitis (Figure 1). US examinations only revealed supportive findings related to acute cholecystitis, including gallbladder wall thickening, biliary stone inside the lumen or in the neck of gallbladder and distension of the gallbladder.

Gallbladder perforation was detected on contrast enhanced MRC images in 3 of our patients and verification of the diagnosis was performed with operation and pathology results (Figure 2). In one of our patients who had a hydatic cyst surgery, a biliary leakage secondary to biliary injury was detected and the same finding was also seen on ERCP images (Figure 3). Heavily T2wMIP image demonstrated abrupt termination of the common bile duct in the patient with choledocholithiasis. Neither heavily T2w-MIP image nor contrast enhanced MRC image could show the bile stone directly. However, contrast enhanced MRC could show the obstruction in contrast material passage through the distal part of the common bile duct (Figure 4). 

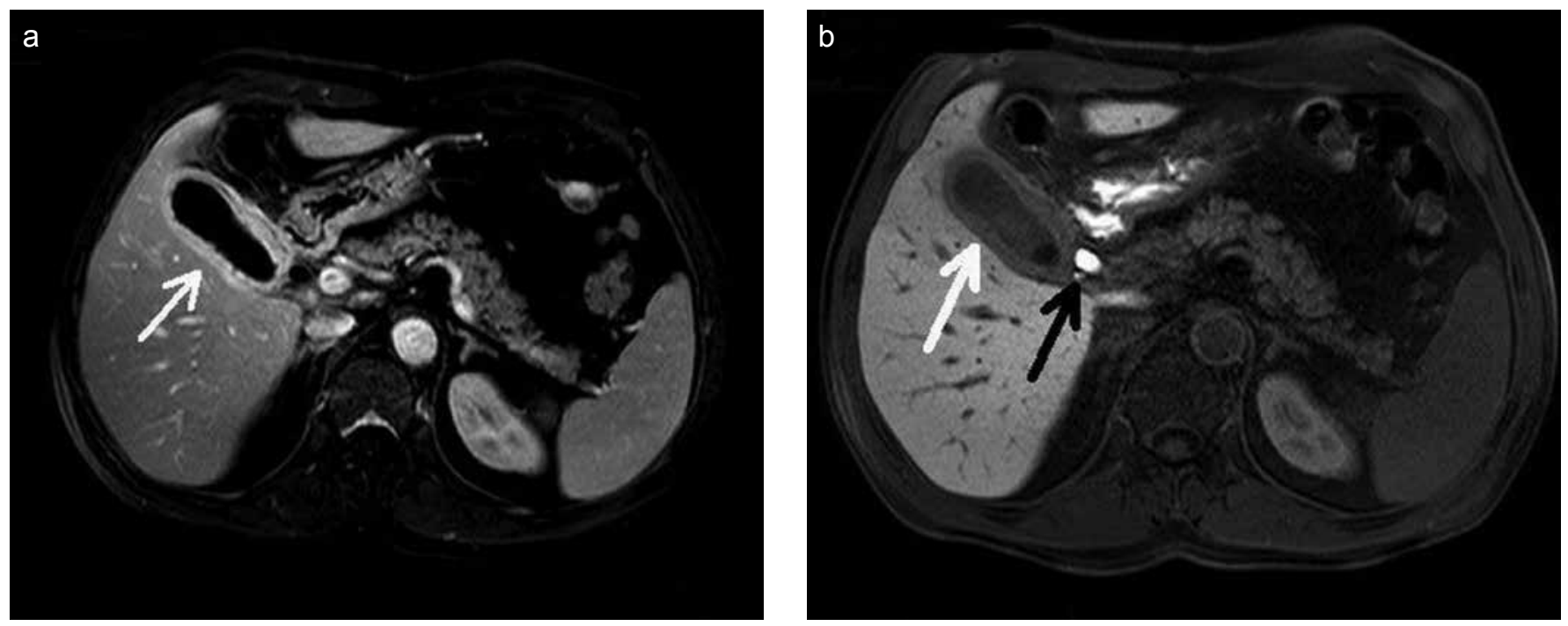

FIG. 1. a, b. A 58 year-old man with acute cholecystitis. Axial contrast enhanced T1w sequence which was obtained in the $4^{\text {th }}$ min after contrast material injection depicts the avid enhancement of gallbladder wall (arrow) (a). Axial contrast enhanced T1w sequence which was obtained in the $120^{\text {th }}$ min after contrast material injection demonstrates that the contrast material did not pass through the gallbladder lumen (white arrow), but the common bile duct was filled with contrast material (black arrow) (b).
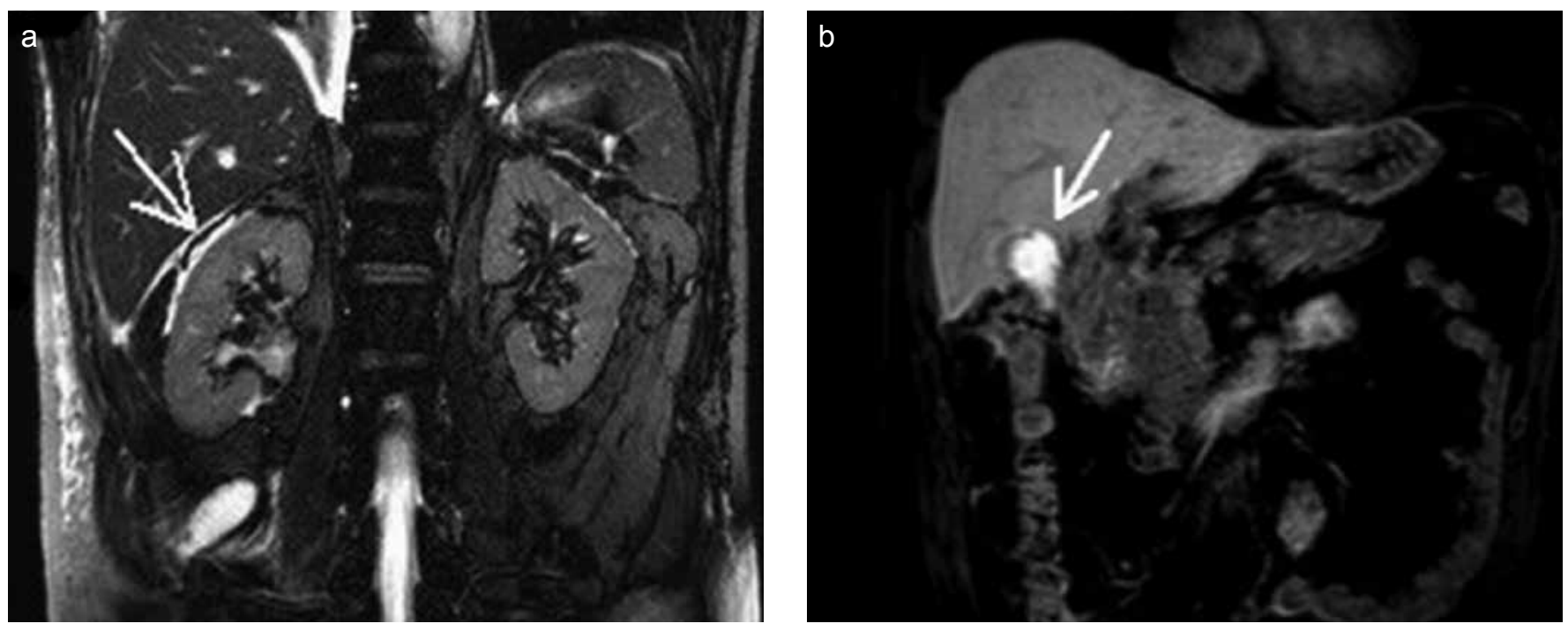

FIG. 2. a, b. A 64 year-old man with gallbladder perforation. T2w-BTFE sequence shows subhepatic fluid collection (arrow) (a). Coronal contrast enhanced T1w sequence which was obtained in the $120^{\text {th }}$ min after contrast material injection shows the leakage of contrast material to the outside of the gallbladder (arrow) (b).

\section{Sensitivity, specificity and accuracy values}

The sensitivity values of T2w-MRCP sequences were between 14 and $25 \%$ for the first reader, while they were between 14 and $60 \%$ for the second. The sensitivity values of contrast enhanced MRC sequences were $100 \%$ for all. The specificity values of T2w-MRCP sequences were between 90 and $92 \%$ for the first reader and between 80 and $87 \%$ for the second reader. The specificity value of the contrast enhanced MRC sequence was $87 \%$ for both readers. The accuracy values of contrast enhanced MRC sequences were significantly higher than those of T2w-MRCP sequences $(\mathrm{p}<0.05)$ (Table 2).

\section{Correlation analysis with gold standard tests}

Contrast enhanced MRC sequences were significantly correlated with the gold standard test results for the first reader, while contrast enhanced MRC and 3D heavily T2w-source images were significantly correlated with the gold standard test results for the second reader $(\mathrm{p}<0.05)$. The other sequences were not correlated significantly with gold standard test re- 

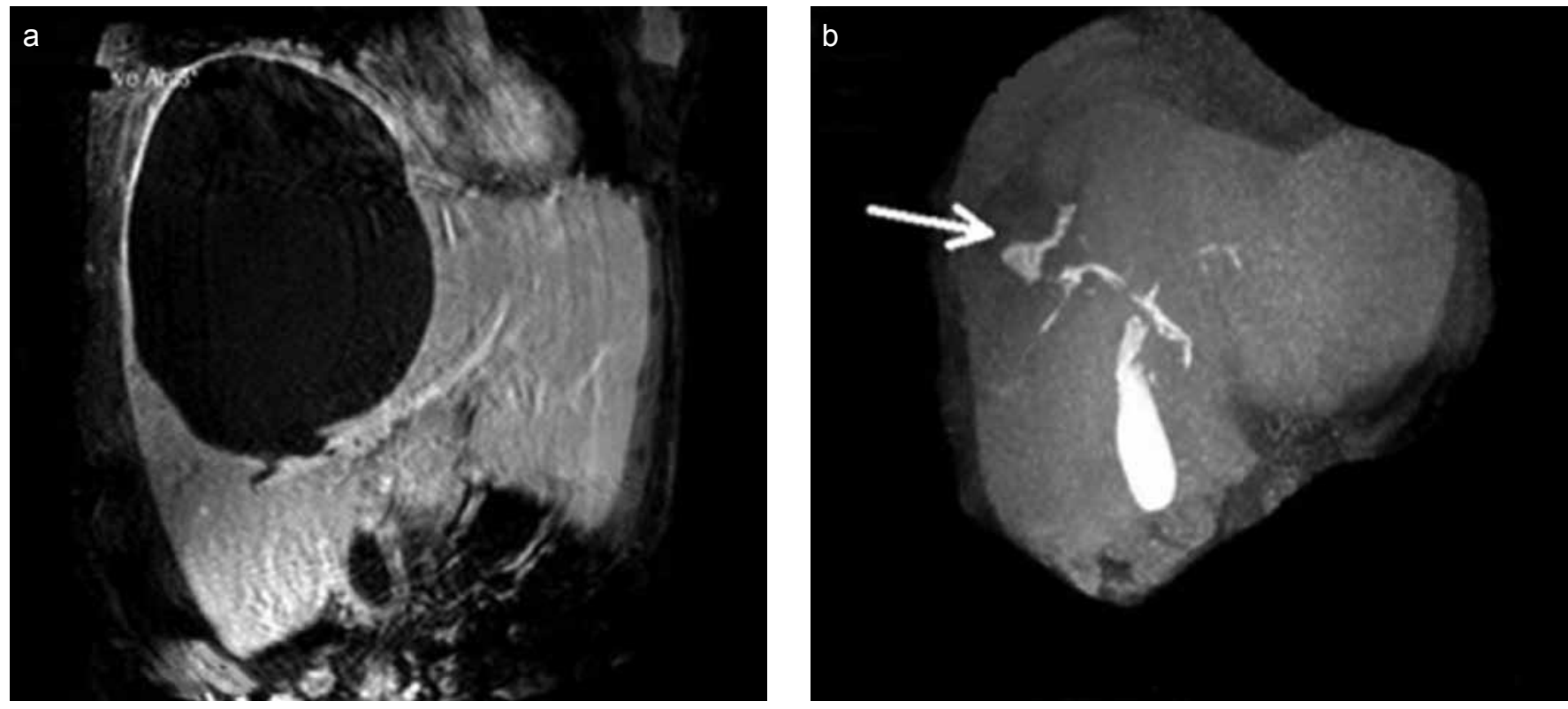

FIG. 3. a, b. Pre and post-operative MR images of a 39 year-old woman with biliary leakage after the hydatid cyst operation. Coronal contrast enhanced T1w image which was obtained before the surgery demonstrates a huge hydatid cyst at the right liver (a). Contrast enhanced T1w-MIP image confirms the leakage of contrast material outside of biliary system (arrow) (b).
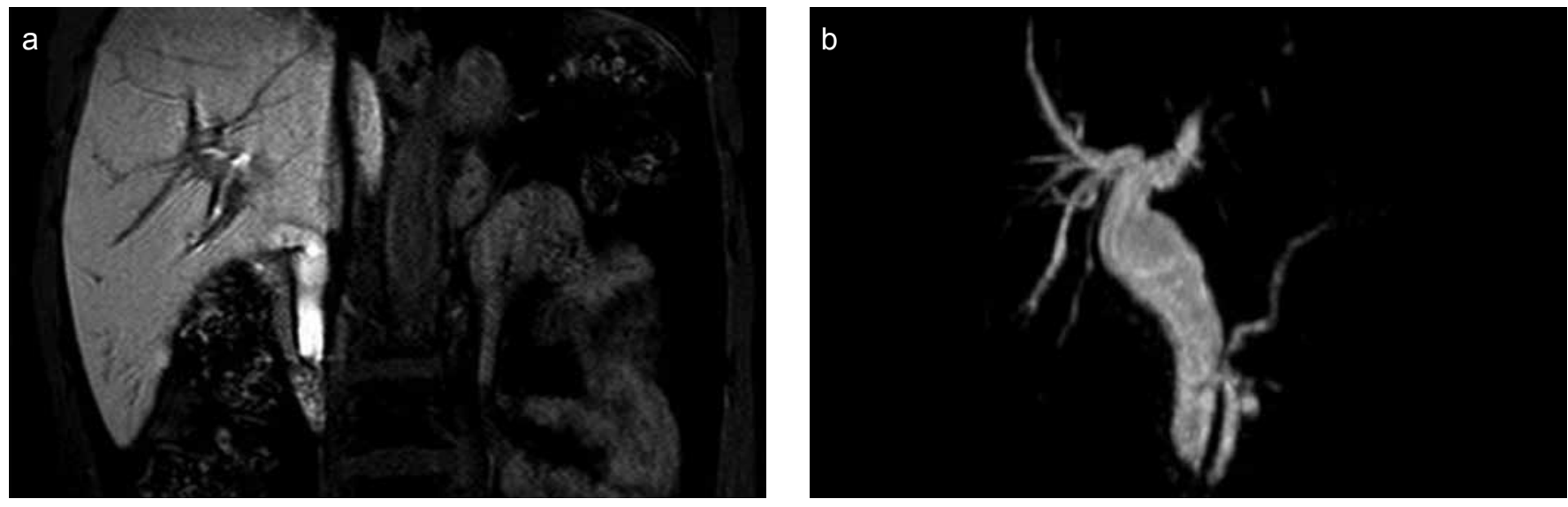

FIG. 4. a, b. Preoperative MR images of a 61 year-old woman with choledocholithiasis. Heavily T2w-MIP image demonstrates abrupt termination of the common bile duct in the patient with choledocholithiasis (a). Contrast enhanced MRC shows the obstruction in contrast material passage through the distal part of the common bile duct (b).

sults. The correlation ratio between the consensus and gold standard tests was the highest, with a ratio of $88 \%$ (Table 3 ).

\section{Reliability analysis}

In interobserver correlation analysis, there was almost perfect correlation between two observers for contrast enhanced MRC sequences $(\mathrm{K}=0.94)$; for the interobserver correlation, $\mathrm{K}$ values were between 0.56 and 0.89 for T2w-MRCP. The intraobserver correlation analysis indicated that $\mathrm{K}$ values for intraobserver correlation were between 0.78 and 0.84 for contrast enhanced MRC sequences, while they were between 0.39 and 0.7 for T2w-MRCP sequences.

\section{DISCUSSION}

The current algorithm for investigating any biliary disorders is to perform US examination since it is easy, non-invasive and does not involve ionizing radiation. In cases where the findings are non-diagnostic, T2w-MRCP should be considered (14). T2w-MRCP highlights the biliary tree, especially with heavily $\mathrm{T} 2 \mathrm{w}$ sequences. However, in some circumstances, T2w-MRCP might be insufficient, especially in the diagnosis of acute cholecystitis biliary leakage and gallbladder perforation $(5,14,15)$. We suggest that contrast enhanced MRC sequences can increase the diagnostic performance of 
TABLE 2. Sensitivity, specificity, and accuracy values of the sequences

\begin{tabular}{|c|c|c|c|c|c|c|c|c|c|}
\hline \multirow[b]{2}{*}{ Sequences } & \multicolumn{3}{|c|}{ Sensitivity (\%) } & \multicolumn{3}{|c|}{ Specificity (\%) } & \multicolumn{3}{|c|}{ Accuracy (\%) } \\
\hline & $1^{\text {st }}$ Observer & $2^{\text {nd }}$ Observer & Mean & $1^{\text {st }}$ Observer & $2^{\text {nd }}$ Observer & Mean & $1^{\text {st }}$ Observer & $2^{\text {nd }}$ Observer & Mean \\
\hline T2w-BTFE & 16.7 & 16.7 & 16.7 & 90 & 80 & 85 & 62.5 & 56.2 & 59.3 \\
\hline T2w-TSE & 20 & 16.7 & 18.3 & 90 & 80 & 85 & 66.7 & 56.2 & 61.4 \\
\hline T2w-SPAIR & 20 & 14.3 & 17.1 & 90 & 80 & 85 & 66.7 & 52.9 & 59.8 \\
\hline 3D-heavily-T2w-sources & 25 & 60 & 42.5 & 91.7 & 86.7 & 89.2 & 65 & 80 & 72.5 \\
\hline Heavily T2w-MIP & 14.3 & 14.3 & 14.3 & 90.9 & 85.7 & 88.3 & 61.1 & 61.9 & 61.5 \\
\hline Axial-CE-T1w & 100 & 100 & 100 & 86.7 & 86.7 & 86.7 & 93.3 & 93.1 & 93.2 \\
\hline Coronal-CE-T1w & 100 & 100 & 100 & 86.7 & 86.7 & 86.7 & 93.3 & 93.3 & 93.3 \\
\hline CE-T1w-MIP & 100 & 100 & 100 & 86.7 & 86.7 & 86.7 & 93.3 & 93.1 & 93.2 \\
\hline Consensus & 100 & 100 & 100 & 86.7 & 86.7 & 86.7 & 93.5 & 93.5 & 93.5 \\
\hline
\end{tabular}

BTFE: balanced turbo field echo; TSE: turbo spin echo; SPAIR: spectral adiabatic inversion recovery; MIP: maximum intensity projection; 3D: three dimensional; CE: contrast enhanced

TABLE 3. The correlations between the sequences and gold standard methods

\begin{tabular}{lcc}
\hline Sequences* & \multicolumn{2}{c}{ Correlation Percent* } \\
& $1^{\text {st }}$ Observer $(\%)^{*}$ & $2^{\text {nd }}$ Observer $(\%)^{*}$ \\
\hline T2w-BTFE* & 26 & 15 \\
T2w-TSE* & 32 & 15 \\
T2w-SPAIR* & 32 & 10 \\
3D heavily T2w-sources* & 34 & $61^{*}$ \\
Heavily T2w-MIP* & 26 & 35 \\
Axial CE-T1w* & $81^{*}$ & $79^{*}$ \\
Coronal CE-T1w* & $83^{*}$ & $81^{*}$ \\
CE-T1w-MIP* & $81^{*}$ & $79^{*}$ \\
Consensus* & $88^{*}$ & $88^{*}$ \\
\hline
\end{tabular}

*: statistically significant ( $\mathrm{p}<0.05)$; BTFE: balanced turbo field echo, TSE: turbo spin echo; SPAIR: spectral adiabatic inversion recovery; MIP: maximum intensity projection; 3D: three dimensional; CE: contrast enhanced

T2w-MRCP. In our study, the sensitivity and accuracy values of contrast enhanced MRC sequences were higher than those of T2w-MRCP. In addition, contrast enhanced MRC findings correlated better with gold standard test results than T2w-MRCP findings. To date, there have only been a limited number of published reports that have focused on the impact of contrast enhanced MRC $(5-9,15,16)$. Among those studies, Krishnan et al. (5) reported that the sensitivity of detection of cystic duct patency for contrast enhanced MRC was $76 \%$ and Salvolini et al. (16) indicated that T2w-MRCP could determine $64 \%$ while contrast enhanced MRC could detect $100 \%$ of the patients with biliary complications after surgery $(5,15)$. Kantarc1 et al. (14) also suggested that contrast enhanced MRC in addition to T2w-MRCP increases the accuracy in detecting bile leaks.
Currently, there are two contrast agents which can be used to perform contrast enhanced MRC: Gd-EOB-DTPA and GdBOPTA. Gd-EOB-DTPA, which has been used in recent years, can diffuse in the extracellular space quickly and is taken up by hepatocytes, similar to Gd-BOPTA. However, the biliary extraction ratio of Gd-EOB-DTPA is about $50 \%$ and significantly higher than Gd-BOPTA (6). This allows a functional evaluation of the biliary system within 20 minutes after administration of the contrast agent in the patients with normal liver function (5,17-19). In recent years, Dahlström et al. (20) compared Gd-BOPTA and Gd-EOB-DTPA enhanced MRC images in terms of biliary tract enhancement in 10 healthy volunteers. In that study, it was emphasized that biliary tract enhancement started earlier and lasted longer with Gd-EOBDTPA (20). The recommended dose of Gd-EOB-DTPA is $0.025 \mathrm{mmol} / \mathrm{kg}$ and is also $1 / 4$ of Gd-BOPTA (10).

In our study, the patients were recommended to fast for about 4-6 hours prior to MRC procedure to enable filling of the gallbladder. As the passage of the contrast agent to the gallbladder could be difficult in postprandial period due to the increase of internal gallbladder pressure, this may reduce the success of contrast enhanced MRC imaging (5). Although some authors recommend the administration of anti-peristaltic drugs (e.g. glucagon), we did not need to use it since we achieved fast sequences and peristaltic artifacts were minimal (21).

Our second important finding was that the correlation values with gold standard tests of contrast enhanced MRC sequences were significantly higher than T2w-MRCP sequences when we consider all of the biliary disorders. Consensus results, which were obtained by evaluating all of the sequences together, showed the highest correlation values with gold standard tests. This indicated that the sequences were complementary to each other and should be evaluated as a whole. Besides, inter and 
intraobserver agreement values of the contrast enhanced MRC sequences were higher than those of T2w-MRCP. These results also showed us that contrast enhanced MRC can provide more reliable results than $\mathrm{T} 2 \mathrm{w}-\mathrm{MRCP}$ images.

The technical parameters of MRC are also very important and can affect the success of the radiologic examination. There were different results in some published reports that focused on choledocholithiasis in the literature. Kats et al. (22) determined the sensitivity of T2 $\mathrm{w}-\mathrm{MRCP}$ as $100 \%$ in choledocholithiasis, whereas Angulo et al. (23) found it to be $50 \%$. We thought that these differences in the literature are related to the technical disparity in those studies and with the variety of biliary diseases in the study groups.

3D heavily $\mathrm{T} 2 \mathrm{w}$-source and post-contrast $\mathrm{T} 1 \mathrm{w}$ acquisitions with small and cubic voxels can be reconstructed to high-resolution multiplanar reformatted and MIP images (23). Nonisotropic acquisition with a larger voxel size has some limitations in the detection of intraductal abnormalities, because small structures that make signal-voids, such as small stones, could be obscured due to the partial volume effect of the intraductal liquid signal intensity. Thus, thin slices should be evaluated carefully in some conditions including the existence of small stones or subtle mural irregularity (23). In our study, the sensitivity, specificity and accuracy values of 3D heavily $\mathrm{T} 2 \mathrm{w}$-source images were higher than those of heavily $\mathrm{T} 2 \mathrm{w}-$ MIP images. The mean sensitivity, specificity and accuracy values of $3 \mathrm{D}$ heavily $\mathrm{T} 2 \mathrm{w}$-source images were $42.5 \%, 89.2 \%$ and $72.5 \%$, respectively, while the sensitivity, specificity and accuracy values of heavily T2w-MIP images were $14.3 \%$, $88.3 \%$ and $61.5 \%$, respectively. In addition, the sensitivity and specificity values of $3 \mathrm{D}$ heavily $\mathrm{T} 2 \mathrm{w}$-source images were the highest among those of all T2w-MRCP sequences.

We think that contrast enhanced MRC sequences could also be helpful in the determination of any possible connection between the liver and peribiliary cysts and bile ducts. MR cholangiography can detect hydatid cysts and demonstrate post-interventional complications including biliary leakage or fistulization, especially on delayed-phase images (24). We did not see any connection between the liver cysts and bile ducts in any of our 5 patients. There were hydatid cysts in 3 of them. However, we also know that we may not visualize the passage of contrast agent to the hydatid cysts, since the internal pressure of hydatid cysts is high, even if those are connected with the bile ducts. Thus, it would not be proper to say that there is no connection with the biliary system for hydatid cysts when we could not see the passage of contrast agent into these cysts on contrast enhanced MRC images. However, Kantarci et al. (25) reported that contrast enhanced MRC is superior in the detection of communication hydatid cyst with biliary system with a sensitivity of $87.4 \%$ and accuracy of $90.5 \%$.
The major drawback of our study is the limited number of patients for each group of biliary diseases. Thus, we could not assess the sensitivity, specificity, and accuracy values separately for each subgroup. More extensive and comprehensive studies are needed for each biliary disease mentioned above. Our second limitation was the need for additional sequences to obtain delayed contrast enhanced MRC sequences. However, we achieved 3 additional sequences in the patients with acute cholecystitis and 2 additional sequences for the remaining patients. Those sequences took about $5 \mathrm{~min}$. In addition, although we detected sensitivity, specificity and accuracy values of each T2w-MRCP sequence separately, we did not evaluate and score all of the T2w-MRCP sequences together. Thus, the last limitation of our study was that we could not exhibit data regarding the sensitivity, specificity and accuracy values of the T2w-MRCP technique. However, we exhibited the sensitivity, specificity and accuracy values of consensus which was obtained by evaluating all of the T2w-MRCP and contrast enhanced MRC sequences together.

In conclusion, the sensitivity, specificity, and accuracy values of Gd-EOB-DTPA enhanced MRC were significantly higher than those of the T2w-MRCP in the diagnosis of biliary diseases. Also, the ratios of correlation with gold standard methods of the contrast enhanced MRC images were significantly higher than the T2w-MRCP images. It would be useful to obtain contrast enhanced MRC sequences in addition to the T2w-MRCP sequences for the diagnosis of biliary diseases.

Ethics Committee Approval: Ethics committee approval was obtained for this prospective study from Yildırım Beyazit University School of Medicine (B.30.2.YBÜ.006.06.01/5).

Informed Consent: Written informed consent was obtained from patients who participated in this study.

Peer-review: Externally peer-reviewed.

Author contributions: Concept - E.O., O.A.; Design - E.O., O.A.; Supervision - H.A.; Resource - O.A.; Materials - E.O., Ş.E.; Data Collection and/or Processing - E.O., Ş.E.; Analysis and/or Interpretation - E.O., Ş.E.; Literature Search - E.O.; Writing - E.O.; Critical Reviews - O.A.

Conflict of Interest: No conflict of interest was declared by the authors.

Financial Disclosure: The authors declared that this study has received no financial support.

\section{REFERENCES}

1. Papanikolaou N, Prassopoulos P, Eracleous E, Maris T, Gogas C, Gourtsoyiannis N. Contrast enhanced magnetic resonance cholan- 
giography versus heavily T2-weighted magnetic resonance cholangiography. Invest Radiol 2001;36:682-6. [CrossRef]

2. Asbach P, Warmuth C, Stemmer A, Rief M, Huppertz A, Hamm $\mathrm{B}$, et al. High spatial resolution T1-weighted MR imaging of liver and biliary tract during uptake phase of a hepatocyte-specific contrast medium. Invest Radiol 2008;43:809-15. [CrossRef]

3. Holzapfel K, Breitwieser C, Prinz C, Rummeny EJ, Gaa J. Contrast-enhanced magnetic resonance cholangiography using gadolinium-EOB-DTPA. Preliminary experience and clinical applications. Radiologe 2007;47:536-44. [CrossRef]

4. Marin D, Bova V, Agnello F, Youngblood R, Midiri M, Brancatelli G. Gadoxetate disodium-enhanced magnetic resonance cholangiography for the noninvasive detection of an active bile duct leak after laparoscopic cholecystectomy. J Comput Assist Tomogr 2010;34:213-6. [CrossRef]

5. Krishnan P, Gupta RT, Boll DT, Brady CM, Husarik DB, Merkle EM. Functional evaluation of cystic duct patency with GdEOB-DTPA MR imaging: an alternative to hepatobiliary scintigraphy for diagnosis of acute cholecystitis? Abdom Imaging 2012;37:457-64. [CrossRef]

6. Akpınar E, Turkbey B, Karcaaltincaba M, Balli O, Akkapulu $\mathrm{N}$, Balas $\mathrm{S}$, et al. Initial experience on utility of gadobenate dimeglumine (Gd-BOPTA) enhanced T1-weighted MR cholangiography in diagnosis of acute cholecystitis. J Magn Reson Imaging 2009;30:578-5. [CrossRef]

7. Kim KW, Park MS, Yu JS, Chung JP, Ryu YH, Lee SI, et al. Acute cholecystitis at T2-weighted and manganese-enhanced T1-weighted MR cholangiography: preliminary study. Radiology 2003;227:580-4. [CrossRef]

8. Aduna M, Larena JA, Martín D, Martínez-Guere-u B, Aguirre I, Astigarraga E. Bile duct leaks after laparoscopic cholecystectomy: value of contrast-enhanced MRCP. Abdom Imaging 2005;30:480-7. [CrossRef]

9. Algin O, Ozlem N, Kilic E, Karaoglanoglu M, Arslan H. GdBOPTA-enhanced MR cholangiography findings in gall bladder perforation. Emerg Radiol 2010;17:487-91. [CrossRef]

10. Seale MK, Catalano OA, Saini S, Hahn PF, Sahani DV. Hepatobiliary-specific MR contrast agents: role in imaging the liver and biliary tree. Radiographics 2009;29:1725-48. [CrossRef]

11. Lee NK, Kim S, Lee JW, Lee SH, Kang DH, Kim GH, et al. Biliary MR imaging with Gd-EOB-DTPA and its clinical applications. Radiographics 2009;29:1707-24. [CrossRef]

12. Landis JR, Brennan P, Silman A. Statistical methods for assessing observer variability in clinical measures. BMJ 1992;304:1491-4. [CrossRef]

13. Landis JR, Koch GC. The measurement of observer agreement for categorical data. Biometrics 1977;33:159-74. [CrossRef]

14. Kantarc1 M, Pirimoglu B, Karabulut N, Bayraktutan U, Ogul $\mathrm{H}$, Ozturk G, et al. Non-invasive detection of biliary leaks us- ing Gd-EOB-DTPA-enhanced MR cholangiography: comparison with T2-weighted MR cholangiography. Eur Radiol 2013;23:2713-22. [CrossRef]

15. Domagk D, Wessling J, Reimer P, Hertel L, Poremba C, Senninger $\mathrm{N}$, et al. Endoscopic retrograde cholangiopancreatography, intraductal ultrasonography, and magnetic resonance cholangiopancreatography in bile duct strictures: a prospective comparison of imaging diagnostics with histopathological correlation. Am J Gastroenterol 2004;99:1684-9. [CrossRef]

16. Salvolini L, Urbinati C, Valeri G, Ferrara C, Giovagnoni A. Contrast enhanced MR cholangiography (MRCP) with GdEOB-DTPA in evaluating biliary complications after surgery. Radiol Med 2012;177:354-68. [CrossRef]

17. Frydrychowicz A, Jedynak AR, Kelcz F, Nagle SK, Reeder SB. Gadoxetic acid-enhanced T1-weighted MR cholangiography in primary sclerosing cholangitis. J Magn Reson Imaging 2012;36:632-40. [CrossRef]

18. Ahuja AT, Griffith JF, Wong KT, Antonio GE, Chu WCW, Ho SSY. Biliary System. In: Ahuja AT, ed. Diagnostic Imaging U1trasound, UK: Amirsys 2007;20-36.

19. Carlos RC, Hussain HK, Song JH, Francis IR. Gadolinium-ethoxybenzyl-diethylenetriamine pentaacetic acid as an intrabiliary contrast agent: preliminary assessment. AJR Am J Roentgenol 2002;179:87-92. [CrossRef]

20. Dahlström N, Persson A, Albiin N, Smedby O, Brismar TB. Contrast enhanced magnetic resonance cholangiography with Gd-BOPTA and Gd-EOB-DTPA in healthy subjects. Acta Radiol 2007;48:362-8. [CrossRef]

21. Halefoğlu AM. Magnetic resonance cholangiopancreatography: a useful tool in the evaluation of pancreatic and biliary disorders. World J Gastroenterol 2007;13:2529-34. [CrossRef]

22. Kats J, Kraai M, Dijkstra AJ, Koster K, Ter Borg F, Hazenberg HJ, et al. Magnetic resonance cholangiopancreaticography as a diagnostic tool for common bile duct stones: a comparison with ERCP and clinical follow-up. Dig Surg 2003;20:32-7. [CrossRef]

23. Angulo P, Pearce DH, Johnson CD, Henry JJ, LaRusso NF, Petersen BT, et al. Magnetic resonance cholangiography in patients with biliary disease: its role in primary sclerosing cholangitis. $J$ Hepatol 2000;33:520-7. [CrossRef]

24. Turkbey B, Akpinar E, Balli O, Tirnaksiz B, Akata D, Akhan $\mathrm{O}$, et al. Clinical applications of gadobenate dimeglumine-enhanced magnetic resonance cholangiography: an expanded pictorial review. Jpn J Radiol 2011;29:3-10. [CrossRef]

25. Kantarci M, Pirimoglu B, Ogul H, Bayraktutan U, Eren S, Aydinli B, et al. Can biliary-cyst communication be predicted by Gd-EOB-DTPA-enhanced MR Cholangiography before treatment for hepatic hydatic disease? Clin Radiol 2013;23:2713-22. 